\title{
Role of street patterns in zone-based traffic safety analysis
}

\author{
GUO Qiang (郭强) ${ }^{1}$, PEI Xin (裴欣 $)^{1,2 *}$, YAO Dan-ya (姚丹亚) $)^{1,2}$, WONG Sze-chun (黄仕进) ${ }^{3}$
}

${ }^{1}$ Department of Automation, Tsinghua University, Beijing, 100084, China

${ }^{2}$ Jiangsu Province Collaborative Innovation Center of Modern Urban Traffic Technologies, SiPaiLou \#2, Nanjing, 210096, China

${ }^{3}$ Department of Civil Engineering, The University of Hong Kong, Pokfulam Road, Hong Kong

\begin{abstract}
Traffic safety has become a growing concern in both developed and developing countries. Although extensive analyses of road segments and intersections located in urban road networks have examined the role of many factors that contribute to the frequency and severity of crashes, the explicit relationship between street pattern characteristics and traffic safety remains underexplored. In this study, using a zone-based Hong Kong database, we use Space Syntax to quantify the topological characteristics of street patterns and investigate the role of street patterns and zone-related factors in zone-based traffic safety analysis. We adopt a joint probability model to analyze crash frequency and severity in an integrated modeling framework and use the maximum likelihood estimation method to estimate the parameters. In addition to the characteristics of street patterns, we consider speed, road geometry, land-use patterns, and temporal factors. We also include vehicle hours as an exposure proxy in the model to make crash frequency predictions. The results indicate that the joint probability model can reveal the relationship between zone-based traffic safety and various other factors, and that street pattern characteristics play an
\end{abstract}


important role in crash frequency prediction.

Key words: street pattern; Space Syntax; joint probability model; crash frequency; crash severity

Resume:

Foundation item: Project (71301083) supported by the National Natural Science Foundation of China; 863 Project (2012AA112305) supported by the National High-Tech Research and Development Program of China; 973 Project (2012CB725405) supported by the National Basic Research Program of China; Project (17208614) supported by the Research Grants Council of the Hong Kong Special Administrative Region, China.

Corresponding author: PEI Xin, Lecturer, PhD; TEL: +86-10-62795043; E-mail: peixin@mail.tsinghua.edu.cn

\section{Introduction}

Traffic safety has become an increasing global concern. In 2010, about 1.24 million people were killed in traffic crashes around the world [1]. It is important to identify and quantify the factors that contribute to crash frequency and severity to improve traffic safety. Although extensive safety analyses of road segments and intersections located in urban road networks have been conducted to examine the role of the various factors that contribute to the frequency and severity of crashes, the explicit relationship between street pattern characteristics and traffic safety has rarely been explored.

RIFAAT et al. [2-5] conducted a series of studies to explore the effects of street patterns on the occurrence and severity of single-vehicle, two-vehicle, pedestrian/bicycle, 
and motorcycle crashes. They chose the City of Calgary in Canada as a case study. They classified street patterns into four categories: gridiron, warped parallel, loops and lollipops, and mixed patterns. They found that limited access patterns were associated with lower crash rates than gridiron patterns. LOVEGROVE and SUN [6] examined the effect of street patterns on road safety at the community level. They examined five road networks to identify road safety: fused grid, commonly used grid, cul-de-sac, 3-way offset, and Dutch sustainable road safety patterns. They found that fused grid and 3-way offset patterns were safer than commonly used grid, cul-de-sac and Dutch sustainable road safety patterns.

These previous studies were limited in that their street pattern classification methods were based on the experience of the researchers, and hence may be prone to subjectivity and uncertainty, due to the unavailability of quantitative street pattern indices. Moreover, their studies examined either the frequency or severity of crashes. The factors that contribute to both crash frequency and severity are important to clarify overall safety performance and improve traffic safety.

In this study, we adopt an innovative street pattern modeling technique known as Space Syntax [7][8], which we use to estimate the street pattern characteristics of 131 urban zones in Hong Kong. We select these urban zones from the traffic analysis zones (TAZ) defined in Travel Characteristics Survey 2002 [9]. The main objective of this study is to explore the effects of street patterns (in terms of their integration in Space Syntax) on both the frequency and severity of crashes using a joint probability regression model [10]. In addition to street patterns, we collect and explore the risk factors (road density [11], 
junction density [12][13], speed [14][15] and land-use patterns [16][17]) and temporal factors [18] of the study zones during 2011 and apply them as control variables.

The remainder of this paper is organized as follows. Section 2 describes the data collection process. Section 3 discusses the structure of the joint probability model. Section 4 summarizes the results of our investigation and offers a discussion. Section 5 presents the conclusions and future research directions.

\section{Data}

\subsection{Street Pattern Measures}

We use Space Syntax to derive several topological characteristics such as connectivity, depth, and integration from the 131 study zones in Hong Kong. Connectivity refers to the number of lines that directly intersect the given line. Depth is defined as the average distance from any other line in terms of the number of links. Integration refers to the accessibility of a whole road network, which is derived by the mean depth.

We define and estimate the various characteristics based on the axial map using lines derived from the traditional maps, with nodes and links representing the space correlation of the streets.

$$
D_{i}=\sum_{d=1}^{s} d \times N_{d}=\left\{\begin{array}{cc}
\text { connectivity } & s=1 \\
\text { local depth } & s=k \\
\text { global depth } & s=l
\end{array}\right.
$$

where $d$ (an integer) denotes the shortest distance from the $i$ th node, $k$ is the number of neighborhoods, $N_{d}$ denotes the number of nodes with the shortest distance $d$, and $l$ denotes the maximum shortest distance. Furthermore, $1<k<l$. $k$ is usually equal to 3 when 
calculating the local depth, and the connectivity is equivalent to the local depth if $k=1$.

We define the mean depth, $\bar{D}_{i}$, as the average distance from the $i$ th node to the other nodes. The formula for calculating the mean depth is

$$
\bar{D}_{i}=D_{i} /(n-1)=\sum_{d=1}^{s} d \times N_{d} /(n-1)=\left\{\begin{array}{cc}
\text { local mean depth } & s=k \\
\text { global mean depth } & s=l
\end{array}\right.
$$

and $D$ is the average of $\bar{D}_{i}$.

The integration value in Space Syntax is calculated as

$$
I=\frac{D_{n}}{2(D-1) /(n-2)}
$$

where $n$ is the number of nodes within the system and

$$
D_{n}=2\left(n\left(\log _{2}((n+2) / 3)-1\right)+1\right) /\left(n^{2}-3 n+2\right)
$$

Integration is correlated with the reciprocal of the mean depth, i.e., the integration value is high when the depth is low and vice versa. Therefore, integration can measure the accessibility of the road network, which is used to represent the street pattern characteristics in this study.

According to the layout of the Hong Kong road network, the study zones can be classified into three graphical categories: grid, deformed grid, and irregular [19]. We choose three samples for each category. Table 1 shows the integration of the road network for each sample zone. The integration factor quantitatively represents the street pattern, which helps describe any type of street pattern.

Table 1 Integration of Nine Samples

G1 G2 G3

D1

D2

D3

I1

I2 


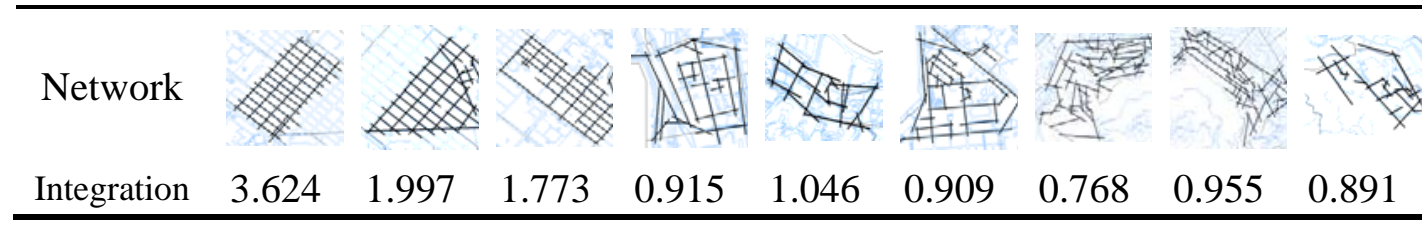

Note: $\mathrm{G}$ = grid; $\mathrm{D}$ = deformed grid; $\mathrm{I}$ = irregular.

\subsection{Crash Data}

We obtain the crash frequency and severity data from the Traffic Information System (TIS), which is maintained by the Hong Kong Police Force and Hong Kong Transport Department. The TIS involves crash-related information, including crash, vehicle and casualty information. In the TIS, crashes are classified into three categories according to severity level: fatal, serious, and slight, as defined by PEI et al. [10]. In this study, we combine the fatal and serious crashes as killed and seriously injured (KSI) crashes that cause serious harm to health and wealth, and attempt to determine the contributory factors that correspond to KSI and slight crashes. Figure 1 shows the distribution of crashes that occurred in the 131 study zones located in the urban area of Hong Kong during 2011. Of the 6,864 crashes that occurred, 884 (12.9\%) were KSI crashes.

\subsection{Speed Data}

Speed is generally considered a factor that determines crash frequency and severity. Speed data collection is thus essential when exploring the effect of speed on traffic safety. In this study, we derive vehicular speeds from 480 GPS-equipped taxis traveling in the roadway network in Hong Kong. Each GPS-equipped taxi transmits instantaneous information about the location, speed, and travel direction to the control center every 30 seconds. We use the Journey Time Survey data obtained from the Department of 
Transportation to verify the validity of the taxi speed data and find that taxi speed is a reasonable proxy for actual travel speed. To reveal the non-linear relationship between speed and safety performance, we classify the average travel speed into four categories: low ( $<20 \mathrm{~km} / \mathrm{h})$, medium (20-40 km/h), medium-high (40-60 km/h), and high (>60 km/h).

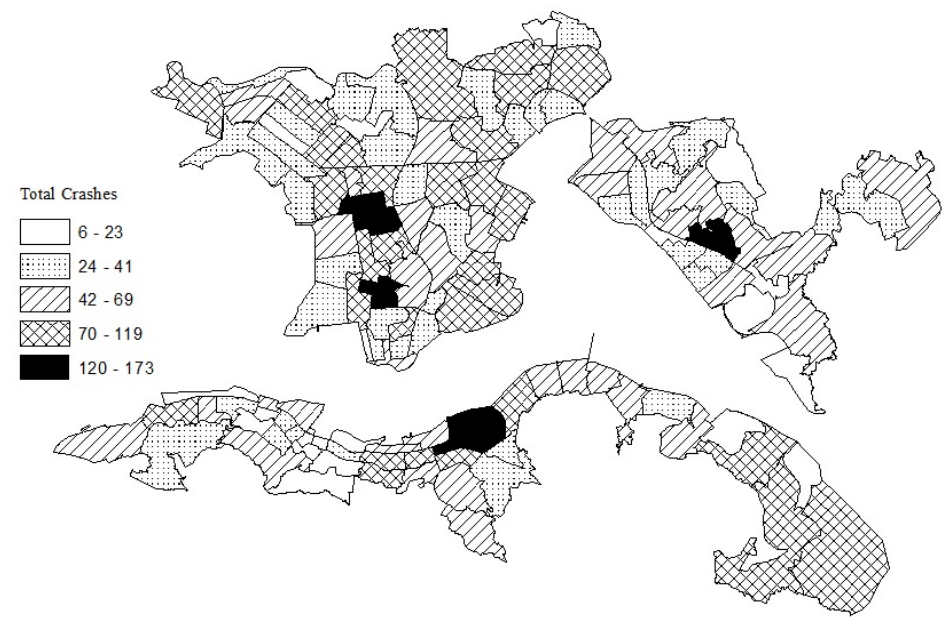

Fig. 1 Crash distributions

\subsection{Exposure}

According to a previous study of the relationship between speed and road safety [20], time exposure measured by vehicle hours $(\mathrm{VH})$ is a more reasonable proxy for exposure in this study.

In terms of the total average annual daily traffic (AADT), traffic volumes are important for calculating exposure. We obtain the AADT data and its temporal and directional multiplicative factors from the Hong Kong Annual Traffic Census (ATC) system, which is maintained by the Transport Department of Hong Kong [21][22]. The ATC system provides the hourly traffic volume for approximately 100 core station roads around Hong Kong. According to a study conducted by WONG and WONG [23, 24], we adopt a linear 
data projection method to combine the AADT and taxi GPS dataset. First, we calculate the scaling factors for each ATC core station. Second, we predict the scaling factor for each analyzed zone according to the spatial correlation of the related core stations. Consequently, we estimate both the hourly traffic spots within each zone and the time exposure as it relates to the last time for each spot, which is supposed to be equal to the update time of the taxi GPS data. We incorporate a logarithmically transformed VH into the joint probability model to explore the effect of exposure time on crash frequency.

\subsection{Summary Statistics of Variables}

To examine the role of the street patterns and zone-related factors that contribute to traffic safety at a more disaggregated level, the database is organized for every 4-hour period: 07:00-11:00 (morning), 11:00-15:00 (noon), 15:00-19:00 (afternoon), 19:00-23:00 (evening), 23:00-03:00 (middle of the night), and 03:00-07:00 (dawn).

Finally, to explore the effect of street patterns on traffic safety, we control various relevant factors in the model, including integration, speed, road geometry, land-use patterns, and temporal factors. In multivariate analysis, it is important to assume that all of the explanatory variables are statistically independent. To eliminate this problem, we calculate the correlations between the explanatory variables. All of the values are less than 0.60 , indicating no evidence of a serious correlation problem. Table 2 illustrates the descriptive statistics of the factors included in the final models.

Table 2 Summary Statistics of Variables

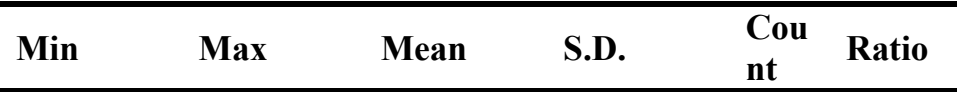




\begin{tabular}{|c|c|c|c|c|c|c|}
\hline \multicolumn{7}{|l|}{ Number of observations=786 } \\
\hline Total crashes & 0 & 53 & 8.733 & 7.720 & & \\
\hline KSI crashes & 0 & 7 & 1.125 & 1.349 & & \\
\hline Integration & 0.479 & 3.624 & 1.146 & 0.428 & & \\
\hline Road density $\left(\mathrm{km} / \mathrm{km}^{2}\right)$ & 21.198 & 109.392 & 50.074 & 14.962 & & \\
\hline $\begin{array}{l}\text { Junction density } \\
\text { (Number of intersections per km) }\end{array}$ & 0.708 & 8.227 & 2.680 & 1.379 & & \\
\hline $\operatorname{Ln}(\mathrm{VH})$ & 5.371 & 13.516 & 11.044 & 1.250 & & \\
\hline Low speed $(0-20 \mathrm{~km} / \mathrm{h})$ & & & & & 295 & $37.5 \%$ \\
\hline Medium speed (20-40 km/h) & & & & & 362 & $46.0 \%$ \\
\hline Medium-high speed (40-60 km/h) & & & & & 115 & $14.6 \%$ \\
\hline High speed (60-80 km/h) & & & & & 14 & $1.8 \%$ \\
\hline 07:00-11:00 & & & & & 131 & $16.7 \%$ \\
\hline 11:00-15:00 & & & & & 131 & $16.7 \%$ \\
\hline 15:00-19:00 & & & & & 131 & $16.7 \%$ \\
\hline 19:00-23:00 & & & & & 131 & $16.7 \%$ \\
\hline 23:00-03:00 & & & & & 131 & $16.7 \%$ \\
\hline 03:00-07:00 & & & & & 131 & $16.7 \%$ \\
\hline Residential & & & & & 462 & $58.7 \%$ \\
\hline Commercial & & & & & 150 & $19.1 \%$ \\
\hline Mixed land & & & & & 102 & $13.0 \%$ \\
\hline Other & & & & & 72 & $9.2 \%$ \\
\hline
\end{tabular}

\section{Method}

We establish a joint probability model [10] to model crash occurrences and severity simultaneously in an integrated modeling framework, and apple the maximum likelihood estimation simulation method to estimate the relevant parameters.

A count data model that takes the form of either a Poisson regression model or a negative binomial regression model is usually applied to explore the effects of related factors on crash frequency. In this study, the accident frequency data is over-dispersed (i.e., the variance of the data is significantly greater than its mean, violating the assumption made in the Poisson regression model). Therefore, we adopt the negative binomial regression model to predict the crash frequency. The formulation of the negative binomial distribution is defined as 


$$
P\left(y_{i}\right)=\frac{\Gamma\left(\varphi+y_{i}\right)}{\Gamma(\varphi) y_{i} !} u_{i}^{\varphi}\left(1-u_{i}\right)^{y_{i}}
$$

where $u_{i}=\varphi /\left(\varphi+\lambda_{i}\right)$ and $\varphi=1 / \alpha$. We define the expected accident frequency $\lambda_{i}$ as a function of the independent variables such that $\lambda_{i}=\exp \left(\theta X_{i}+\ln (\mathrm{VH})\right)$, where $X_{i}$ is the vector for the independent variables, $\theta$ is the vector for the estimable coefficients, and $\mathrm{VH}$ is the time exposure.

Two severity levels, KSI and slight, are quantified in this study. We establish a binomial logit model based on the frequency of KSI crashes, which we use as the dependent variable for the crash severity prediction.

Let $k_{i}^{\mathrm{KSI}}$ denote the number of crashes at the KSI severity level. We assume that $k_{i}^{\mathrm{KSI}}$ follows a binomial distribution with parameters $\pi_{i}^{\mathrm{KSI}}$ and $y_{i}$, as $k_{i}^{\mathrm{KSI}} \sim \operatorname{Binomial}\left(\pi_{i}^{\mathrm{KSI}}, y_{i}\right)$ with the probability function

$$
P\left(k_{i}^{\mathrm{KSI}} \mid y_{i}\right)=\left(\begin{array}{c}
y_{i} \\
k_{i}^{\mathrm{KSI}}
\end{array}\right)\left(\pi_{i}^{\mathrm{KSI}}\right)^{k_{i}^{\mathrm{KSI}}}\left(1-\pi_{i}^{\mathrm{KSI}}\right)^{y_{i}-k_{i}^{\mathrm{KSI}}}
$$

where $y_{i}$ represents the total crashes and $\pi_{i}^{\mathrm{KSI}}$ represents the probability of KSI crashes.

We apply a logit model to estimate the associations between the binomial probability $\pi_{i}^{\mathrm{KSI}}$ and the independent variables, which are defined as

$$
\log i t\left(\pi_{i}^{\mathrm{KSI}}\right)=\log \left(\frac{\pi_{i}^{\mathrm{KSI}}}{1-\pi_{i}^{\mathrm{KSI}}}\right)=\beta^{\mathrm{KSI}} X_{i}
$$

where $\beta^{\mathrm{KSI}}$ is the vector for the corresponding coefficients.

Consequently, the joint probability function for the frequency and severity of crashes is defined as 


$$
\begin{aligned}
P\left(y_{i}, k_{i}^{\mathrm{KSI}}\right)= & P\left(y_{i}\right) \times P\left(k_{i}^{\mathrm{KSI}} \mid y_{i}\right) \\
& =\frac{\Gamma\left((1 / \alpha)+y_{i}\right)}{\Gamma(1 / \alpha) y_{i} !}\left(\frac{1 / \alpha}{(1 / \alpha)+\exp \left(\theta X_{i}+\ln (\mathrm{VH})\right)}\right)^{1 / \alpha}\left(\frac{\exp \left(\theta X_{i}+\ln (\mathrm{VH})\right)}{(1 / \alpha)+\exp \left(\theta X_{i}+\ln (\mathrm{VH})\right)}\right)^{y_{i}} \\
& \times\left(\begin{array}{c}
y_{i} \\
k_{i}^{\mathrm{KSI}}
\end{array}\right)\left(\frac{\exp \left(\beta^{\mathrm{KSI}} X_{i}\right)}{1+\exp \left(\beta^{\mathrm{KSI}} X_{i}\right)}\right)^{k_{i}^{\mathrm{KSI}}}\left(\frac{1}{1+\exp \left(\beta^{\mathrm{KSI}} X_{i}\right)}\right)^{y_{i}-k_{i}^{\mathrm{KSI}}}
\end{aligned}
$$

\section{Results and discussion}

We use the maximum likelihood estimation simulation method to estimate the relevant parameters. Table 3 presents the estimation results of the joint probability model.

\begin{tabular}{|c|c|c|c|c|}
\hline & \multicolumn{2}{|c|}{ Crash frequency } & \multicolumn{2}{|c|}{ Crash severity } \\
\hline & $\beta$ & Sig. & $\beta$ & Sig. \\
\hline \multicolumn{5}{|l|}{ Number of observations $=786$} \\
\hline Constant & $-8.276^{* *}$ & 0.000 & $-1.281^{* *}$ & 0.000 \\
\hline Integration & $-0.144^{*}$ & 0.072 & -0.007 & 0.940 \\
\hline Road density & $-0.012^{* *}$ & 0.000 & -0.003 & 0.237 \\
\hline Junction density & $0.174^{* *}$ & 0.000 & -0.043 & 0.254 \\
\hline High speed (60-80 km/h) & $0.713^{* *}$ & 0.007 & 0.197 & 0.563 \\
\hline Medium-high speed $(40-60 \mathrm{~km} / \mathrm{h})$ & $-0.192^{*}$ & 0.077 & $0.279^{*}$ & 0.054 \\
\hline Medium speed $(20-40 \mathrm{~km} / \mathrm{h})$ & $-0.294^{* *}$ & 0.000 & 0.134 & 0.116 \\
\hline \multicolumn{5}{|l|}{ Low speed $(0-20 \mathrm{~km} / \mathrm{h})$} \\
\hline 07:00-11:00 & -0.087 & 0.461 & -0.183 & 0.259 \\
\hline 11:00-15:00 & $-0.211^{*}$ & 0.072 & $-0.423^{* *}$ & 0.010 \\
\hline 15:00-19:00 & $-0.247^{* *}$ & 0.035 & $-0.439^{* *}$ & 0.007 \\
\hline 19:00-23:00 & -0.173 & 0.140 & $-0.438^{* *}$ & 0.009 \\
\hline 23:00-03:00 & $-0.392^{* *}$ & 0.001 & $-0.432^{* *}$ & 0.022 \\
\hline \multicolumn{5}{|l|}{ 03:00-07:00 } \\
\hline Commercial & $-0.439^{* *}$ & 0.000 & $-0.246^{* *}$ & 0.047 \\
\hline Mixed land & $-0.399^{* *}$ & 0.000 & -0.096 & 0.338 \\
\hline Other & $-0.517^{* *}$ & 0.000 & -0.217 & 0.207 \\
\hline Residential & (control) & & & \\
\hline \multicolumn{5}{|l|}{ Over-dispersion parameter } \\
\hline$A$ & $0.505^{* *}$ & & & \\
\hline \multicolumn{5}{|l|}{ Goodness-of-fit } \\
\hline$\overline{\text { Log-likelihood }}$ & -5080 & & & \\
\hline P-value & $<0.0001$ & & & \\
\hline
\end{tabular}
In general, the goodness-of-fit statistics confirm that the model fits the data well.

Table 3 Joint Probability Model Results

**Statistically significant at the $5 \%$ level 
*Statistically significant at the $10 \%$ level

The main objective of this study is to determine how street pattern characteristics related to integration are associated with crash frequency and severity. As shown in Table 3, integration is negatively correlated with crash frequency $[\beta=-0.144, p<0.10]$ at the $10 \%$ significance level. As shown in Table 1, the higher integration value implies a more integrated system and a better spatial accessibility and visual field. These findings are helpful in avoiding crash occurrences and thereby decreasing the crash frequency.

In addition to integration, other road characteristics, particularly road density, are negatively correlated with the crash frequency $[\beta=-0.012, p<0.01]$ at the $5 \%$ significance level, indicating that the higher the road density, the lower the crash frequency. This finding may be also related to road accessibility. As expected, junction density is positively correlated with the crash frequency $[\beta=0.174, p<0.01]$ at the $5 \%$ significance level. Consistent with previous studies by PRIYANTHA WEDAGAMA et al. [12] and XU and HUANG [13], our results show that increased junction density is significantly associated with a higher total crash frequency. This finding is reasonable because intersections are hazardous locations due to the number of potential conflict points and unexpected driver behavior.

In addition to road characteristics, we explore the effect of average speed on crash frequency and severity and consider four speed levels: low ( $<20 \mathrm{~km} / \mathrm{h})$, medium (20-40 $\mathrm{km} / \mathrm{h})$, medium-high $(40-60 \mathrm{~km} / \mathrm{h})$, and high $(>60 \mathrm{~km} / \mathrm{h})$. The average speed shows a U-shaped effect. Compared with low speed, medium $[\beta=-0.294, p<0.01]$ and medium-high 
speeds [ $\beta=-0.192, p<0.10]$ are negatively correlated with the crash frequency at the $5 \%$ and $10 \%$ significance levels, respectively. High speed is positively correlated with the crash frequency $[\beta=0.713, p<0.01]$ at the $5 \%$ significance levels. Consistent with previous studies by GLADHILL and MONSERE [14] and GARBER and GADIRAU [25], our results show that higher average speeds (low to medium-high) are associated with a lower accident frequency. Favorable driving conditions may account for the reduction in crash frequency at higher average speeds. The roads inside the communities are higher-grade roads, the road infrastructures are relatively perfect, and the road environment is favorable for driving, which results in better vehicle control and decreases the crash frequency. However, as speeds increase, the result reverses due to the decreased time available for driver reaction and corresponding defensive vehicle maneuvers. In terms of crash severity, all three of these levels of speed increase crash severity, but only the result for medium-high speed is statistically significant $[\beta=0.279, p<0.10]$ at the $10 \%$ significance level; the results for the other speeds are statistically insignificant. This finding is consistent with previous studies by HAUER [15] and NATIONAL RESEARCH COUNCIL [26]. The higher crash severity is a result of the higher vehicle speeds, which translate to larger impact forces in collisions.

We consider four land-use patterns to identify their influence on crash frequency and severity: residential areas, commercial areas, mixed-land areas, and other areas. Compared with residential areas, the likelihood of crashes occurring in commercial areas $[\beta=-0.439, p<0.01]$, mixed-land areas $[\beta=-0.399, p<0.01]$, and other areas $[\beta=-0.517$, $p<0.01]$ in Hong Kong is significantly lower and at the 5\% significance level. In terms of 
crash severity, commercial areas $[\beta=-0.246, p<0.05]$ are significantly safer than residential areas at the $5 \%$ significance level. Contrary to the findings of previous studies by KIM et al. [16] and OUYANG and BEJLERI [17], we find that the presence of additional overpasses may account for the crash frequency reductions in commercial and mixed-land areas of Hong Kong. As shown in Figure 2, these overpasses force pedestrians to cross the road at points separated from vehicles, thereby ensuring pedestrian safety. Moreover, in most of the residential areas in Hong Kong, ground-floor shops are popular and attractive to surrounding residents (as shown in Figure 3), inducing more pedestrian involvement in crashes.

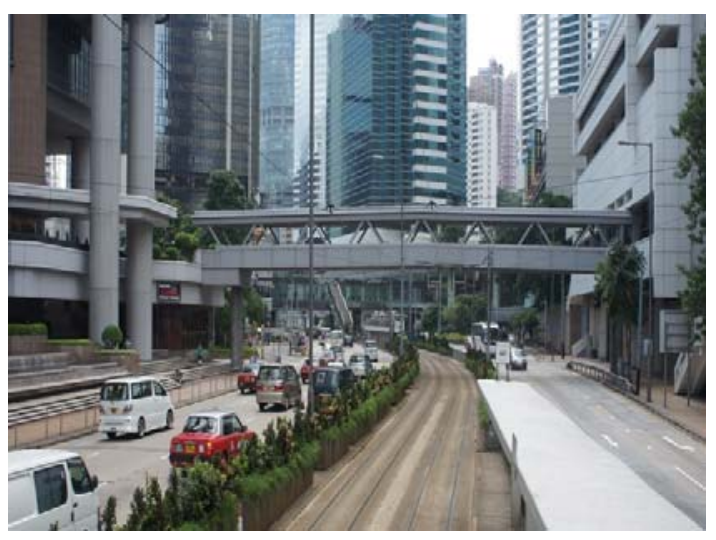

Fig. 2 Commercial area in Hong Kong

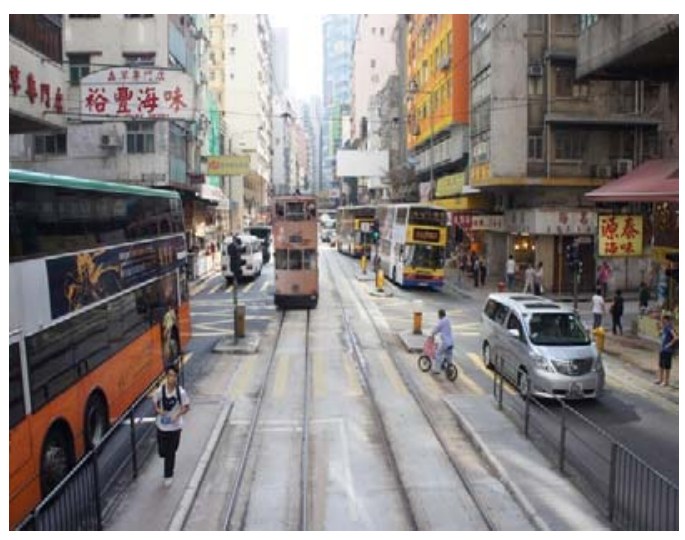

Fig. 3 Residential area in Hong Kong

We include the temporal factor in the model to represent the crash heterogeneity at different times of day. The crash rate and level of KSI risk are higher at dawn than they are at other times of the day. According to LI et al. [18], this is probably a result of the dangerous behavior of drivers at night, such as driving while fatigued, aggressive driving, and driving under the influence of alcohol.

\section{Conclusions}


This study explores the role of the topological characteristics of street patterns in zone-based traffic safety analysis. We derive the integration index via Space Syntax and use it to represent the structures of the street patterns within each TAZ. We apply a joint probability model to estimate crash frequency and severity in an integrated modeling framework. We find that street pattern characteristics play an important role in zone-based traffic safety analysis. Crash severity is significantly related to integration, road density, junction density, average speed, land-use patterns, and temporal factors. With respect to crash severity, commercial areas are associated with slighter crash severity and increased speed is significantly associated with an increase in crash severity.

Future studies should consider the spatial correlation between zones in their models. Other related factors such as detailed social-economic factors may help to further analyze crash frequency and severity.

\section{Acknowledgements}

This research was jointly supported by grants from the National Natural Science Foundation of China (No. 71301083), the National Basic Research Program of China (973 Project; No. 2012CB725405), the National High-Tech Research and Development Program of China (863 Project; No. 2012AA112305), and the Research Grants Council of the Hong Kong Special Administrative Region, China (No. 17208614). We thank Concord Pacific Satellite Technologies Limited and Motion Power Media Limited for providing the GPS taxi data for the research work that is reported in this paper.

\section{References}


[1] WHO. Global Status Report on Road Safety: Time for Action [R]. Geneva, 2013.

[2] RIFAAT S M, TAY R, DE BARROS A G, CHOI J S, PARK D J. Logistic model of injury risks in single vehicle crashes in urban neighborhoods [J]. Journal of Advanced Transportation, 2011, 45(3): 186-195.

[3] RIFAAT S M, TAY R. Effects of street pattern on injury risks in two-vehicle crashes [J]. Transportation Research Record, 2009, 2102: 61-67.

[4] RIFAAT S M, TAY R, DE BARROS A. Effect of street pattern on the severity of crashes involving vulnerable road users [J]. Accident Analysis and Prevention, 2011, 43(1): 276-283.

[5] RIFAAT S M, TAY R, DE BARROS A. Severity of motorcycle crashes in Calgary [J]. Accident Analysis and Prevention, 2012, 49: 44-49.

[6] LOVEGROVE G R, SUN J. Using community-based macrolevel collision prediction models to evaluate safety level of neighborhood road network patterns [C]. Transportation Research Board 89th Annual Meeting, Washington, DC, 2010, Paper \#10-0535.

[7] HILLIER B, HANSON J. The social logic of space [M]. UK: Cambridge University Press, 1984.

[8] HILLIER B. Space is the machine: A configurational theory of architecture [M]. UK: Cambridge University Press, 1996.

[9] TRANSPORT DEPARTMENT. Travel Characteristics Survey 2002 Final Report [R]. Hong Kong SAR Government, 2003.

[10] PEI Xin, WONG S C, SZE N N. A joint-probability approach to crash prediction models [J]. Accident Analysis and Prevention, 2011, 43(3): 1160-1166.

[11] WANG Xue-song, TREMONT P J, CHEN Xiao-hong, JIN Yu, ABDEL-ATY M. Macrolevel model development for safety assessment of road network structures [J]. Transportation Research Record, 2012, 2280: 100-109.

[12] PRIYANTHA WEDAGAMA D M, BIRD R N, METCALFE A V. The influence of urban land-use on non-motorised transport casualties [J]. Accident Analysis and Prevention, 2006, 38(6): 1049-1057.

[13] XU P P, HUANG H L. Modeling crash spatial heterogeneity: Random parameter versus geographically weighting [J]. Accident Analysis and Prevention, 2015, 75: 16-25.

[14] GLADHILL K, MONSERE C M. Exploring traffic safety and urban form in Portland, Oregon [J]. Transportation Research Record, 2012, 2318: 63-74.

[15] HAUER E. Speed and safety [J]. Transportation Research Record, 2009, 2103, 10-17.

[16] KIM K, PANT P, YAMASHITA E Y. Accidents and accessibility: Measuring the influences of demographic and land use variables in Honolulu, Hawaii [J]. Transportation Research Record, 2010, 2147: 9-17.

[17] OUYANG Y, BEJLERI I. Geographic information system-based community-level method to evaluate the influence of built environment on traffic crashes [J]. Transportation Research Record, 2014, 2432: 124-132. 
[18] LI Y C, SZE N N, WONG S C. Spatial-temporal analysis of drink driving patterns in Hong Kong [J]. Accident Analysis and Prevention, 2013, 59: 415-424.

[19] LI Jun. Model and analyze Hong Kong street network using Space Syntax theory [M]. Shandong: Qingdao University, 2007. (In Chinese.)

[20] PEI Xin, WONG S C, SZE N N. The roles of exposure and speed in road safety analysis [J]. Accident Analysis and Prevention, 2012, 48: 464-471.

[21] LAM W H K, HUNG W T, LO H K, LO H P, TONG C O, WONG S C, YANG H. Advancement of the annual traffic census in Hong Kong [C]. Proceedings of the Institution of Civil Engineers, Transport, 2003, 156: 103-115.

[22] TONG C O, HUNG W T, LAM W H K, LO H K, LO H P, WONG S C, YANG H. A new survey methodology for the annual traffic census in Hong Kong [J]. Traffic Engineering and Control, 2003, 44: 214-218.

[23] WONG W, WONG S C. Systematic bias in transport model calibration arising from the variability of linear data projection [J]. Transportation Research Part B, 2015, 75: $1-18$.

[24] WONG W, Wong S C. Network topological effects on the macroscopic Bureau of Public Roads function [J]. Transportmetrica A: Transport Science, under review.

[25] GARBER N J, GADIRAU R. Speed variance and its influence on crashes [R]. AAA Foundation for Traffic Safety, 1988.

[26] NATIONAL RESEARCH COUNCIL. Highway safety manual [M]. USA: American Association of State Highway and Transportation Officials, Washington, DC, 2010. 